\title{
Study on Vision based On-machine Micro Tool Measurement Method Xiaoru LIU ${ }^{1, a}$, Xi ZHANG ${ }^{1,2, b^{*} \text {,Bo SHANG }}{ }^{1, c}$ and Xianliang $\mathrm{CHI}^{1, d}$

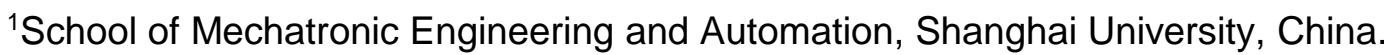 \\ ${ }^{2}$ Shanghai Key Laboratory of Intelligent Manufacturing and Robotics, China. \\ axrliudy@163.com, bxizhang@shu.edu.cn, 'shangbo120812@sina.com, ${ }^{d}$ chiasdf@163.com
}

Keywords: Micro tool inspection, Dimension measurement, Radial runout, Machine vision.

\begin{abstract}
In order to inspect the condition of micro tools automatically and accurately in the micro milling process, a vision based on-machine measurement method is studied in this paper. The proposed methods can measure tool dimension, spindle radial runout and acquire the microscopic image with full depth of focus from both radial and axial direction. Based on the proposed method, a dedicated micro tool measuring system was established, which consists of on-machine measurement unit, control box and software. The measurement experiments on machine tool verified that the proposed methods and the developed measurement system can fulfill the needs of industrial applications.
\end{abstract}

\section{Introduction}

In recent years, with the rapid development of micro/nano science and technology, the miniaturization of mechanical parts with its small size, high precision, stable and reliable, and high sensitivity and other characteristics, have increasingly become one of the future demand for the development of science and technology [1]. Micro milling with conventionally shaped tools has gained increasing importance to meet this demand due to its flexibility to fabricate complex 3D shape with variety of engineering materials. As the important component of micro milling, micro tools are characterized by small size $(0.01 \mathrm{~mm}-1 \mathrm{~mm})$, super hardness and frangibility. The tool failure including wear and breakage may seriously affect the quality of the micro machining product, and even cause damage to machine tool. However, the micro tools are too tiny to be observed directly by the operators. It is necessary to measure tool dimension and monitor tool conditions in order to guarantee the quality and efficiency of micro machining.

The traditional tools condition inspection methods include indirect and direct measurement. Indirect method is to use the tool wear state of different working parameters reflects the degree of tool wear relative variable, which are commonly used method of cutting force test, acoustic emission testing technique, vibration signal test, current signal measurement, etc.[2,3]. The indirect method can implement on-line detection, which didn't affect the machining process. But too many interference factors will interfere the detection especially for the micro tools and the precise result cannot be guaranteed. Direct method can identify the blade appearance, surface quality and geometry changes intuitively. At present the main methods include radiographic test, optical measurement method, and computer image processing method [4]. Due to the tiny size and complicated structure of micro tools, it is an appropriate inspection solution to take and analyze the microscopic image of micro tools. Currently vision based micro tool measurement system such as dynavision [5], however, can only take the images of micro tools from radial direction with limited depth of focus. In this paper, a dedicated vision based on-machine measurement system for inspection of micro tools was studied, which can measure tool dimension, spindle radial runout and acquire the microscopic image with full depth of focus from both radial and axial direction. The paper is organized as follows. First, the developed measurement system is described. Then the measurement method and experimental results for each function is given in details. The last section summarizes the proposed micro tool inspection method 


\section{Micro Cutting Tools Measurement System}

In order to ensure the accuracy and stability of the micro cutting tools measurement, a dedicated vision based on-machine measurement system was designed in this article, as shown in Fig. 1, which include on-machine unit, control box and the software. The on-machine unit is compact to be mounted on the worktable, which consists of CCD camera, telecentric lens and lighting source. The control box was used to supply power and control signals. The software was developed by Visual C++ to implement the function of automatic dimension measurement, spindle radial runout measurement and tool surface observation, which are explained below in details.

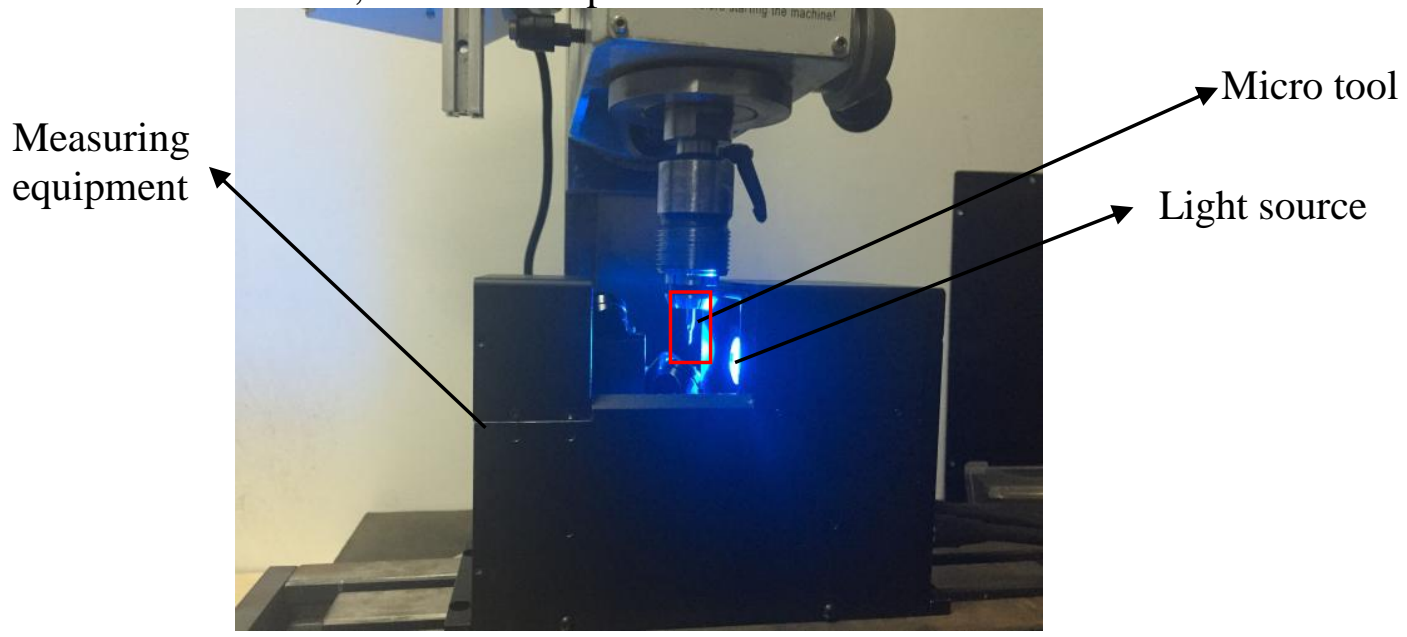

Fig. 1 Vision based On-machine micro tool inspection measurement system

\section{Automatic Dimension Measurement}

In this paper a measurement method was proposed to measure the diameter and length of micro tools based on machine vision automatically. The multiple image of a micro tool with back lighting are taken while the spindle is rotating. The concrete step is to synthesize the image of maximum rotating envelope of the milling cutter from image sequence. Next the contour on this image is analyzed in order to measure the tool dimension automatically. The algorithm is given below.

Generally, the tool contour consists of lines and circular arcs or elliptic arcs. Therefore, the first step is to segment the tool contour and judge contour type. A large number of different contour definitions have been proposed over the years. A very good evaluation of many polygonal approximation methods has been carried out by Rosin. In both approximation cases, Ramer algorithm have the best results for segmenting the contour type [6].The Ramer algorithm is applied to perform a recursive subdivision of the contour until the resulting line segments have a maximum distance to the respective contour segments that is lower than a user-specified threshold $d_{\text {max }}$. Fig.2 illustrates how the Ramer algorithm works. Then each pair of adjacent line segments was checked to see whether it can be better approximated by an ellipse (or, alternatively, a circle). After examining all pairs of line segments, line type is distinguished and the type of the tool can be determined.
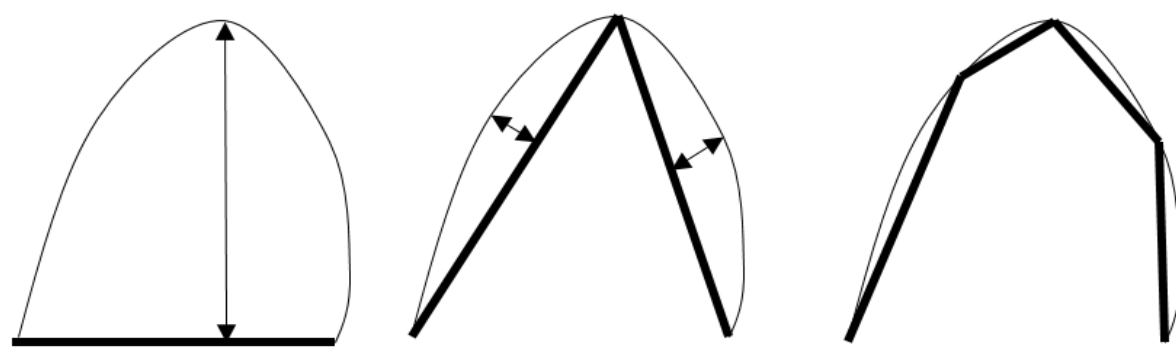

Fig. 2 The principle of Ramer algorithm for contour segementation 
Finally the basic sizes of micro tool, which mainly include the radius and the clamping length in machine tool spindle is calcuated and marked on the image. Fig. 3 shows the dimensions of two typical micro tools.

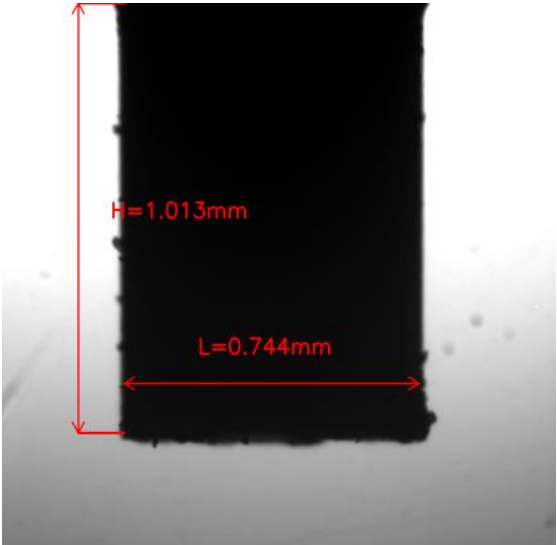

(a) Hexagan end mill

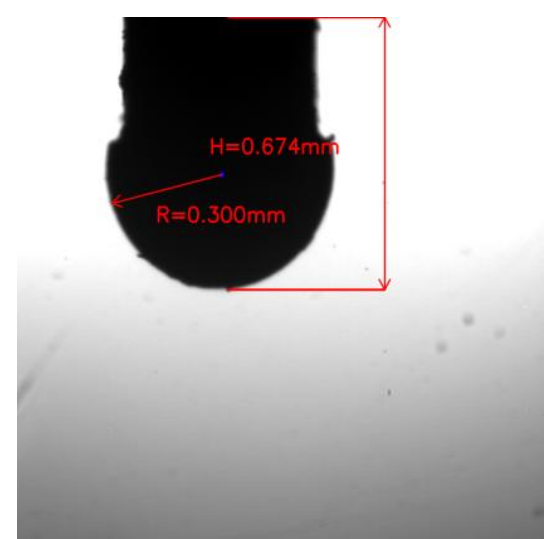

(b) Ball end mill

Fig. 3 The results of micro tools dimension measurement

\section{Radial Runout Test of Machine Tool}

How to detect the radial runout error of the micro machine tool is very important for processing of precision micro parts [7].In this article, a new method based on machine vision is proposed to detect the spindle radial runout.

First of all, a standard cylindrical bar with known diameter of $1 \mathrm{~mm}$ was mounted on the spindle, whose image was taken with back lighting under the spindle stationary state, as shown in Fig. 4 a. Secondly the its image was captured again when the micro machine tool spindle rotates with a normal operation speed. Then the image of the maximum rotating envelope of the standard bar is taken, as shown in image Fig. 4 b. Finally by comparing the difference of contour on Fig $4 \mathrm{a}$ and $\mathrm{b}$, the radial runout of spindle can be calculated. An example of measurment results was shown in Fig. $4 \mathrm{c}$.

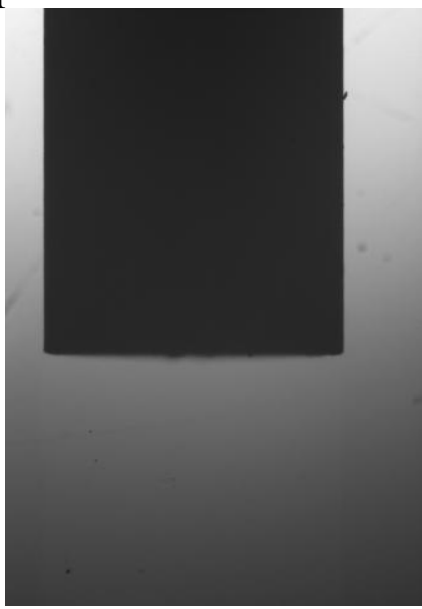

(a) Spindle stationary state

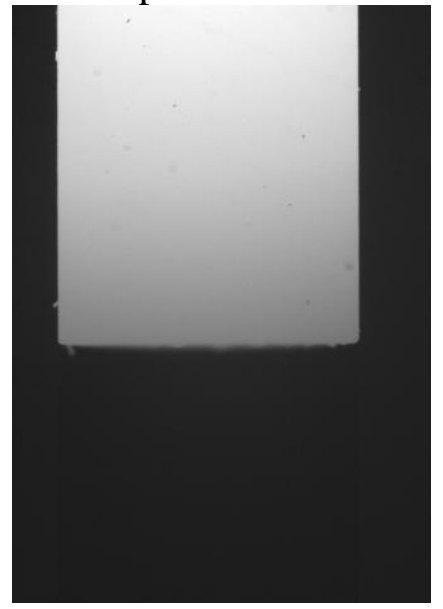

(b) Spindle rotating

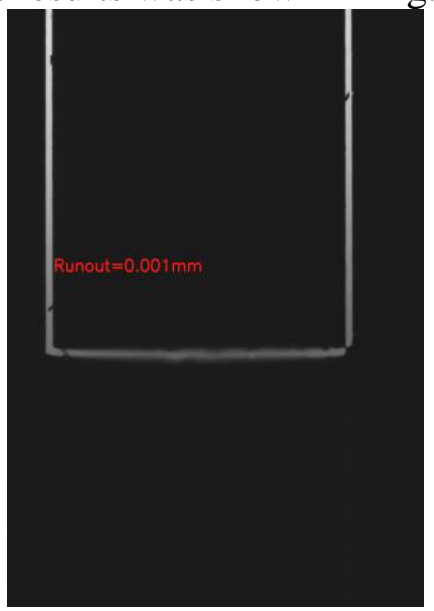

(c) Results of runout

Fig. 4 The process of radial runout inspection

\section{Radial Surface Observation of Micro Tools}

Due to the limited depth-of-focus of optical lenses in CCD devices, it is often not possible to get an image that contains all relevant objects in focus. In an image captured by this device, only those objects within the depth of field are focused, while other objects are blurred [8]. Therefore, the same view point under different focal settings needs to be acquired and then fuse the images, as shown in Fig. 5. The aim of image fusion algorithm is to integrate complementary and redundant information 
from multiple images to create a composite that contains a better description of the scene than any of the individual source images [9]. This fused image will contain all the relevant objects in focus.

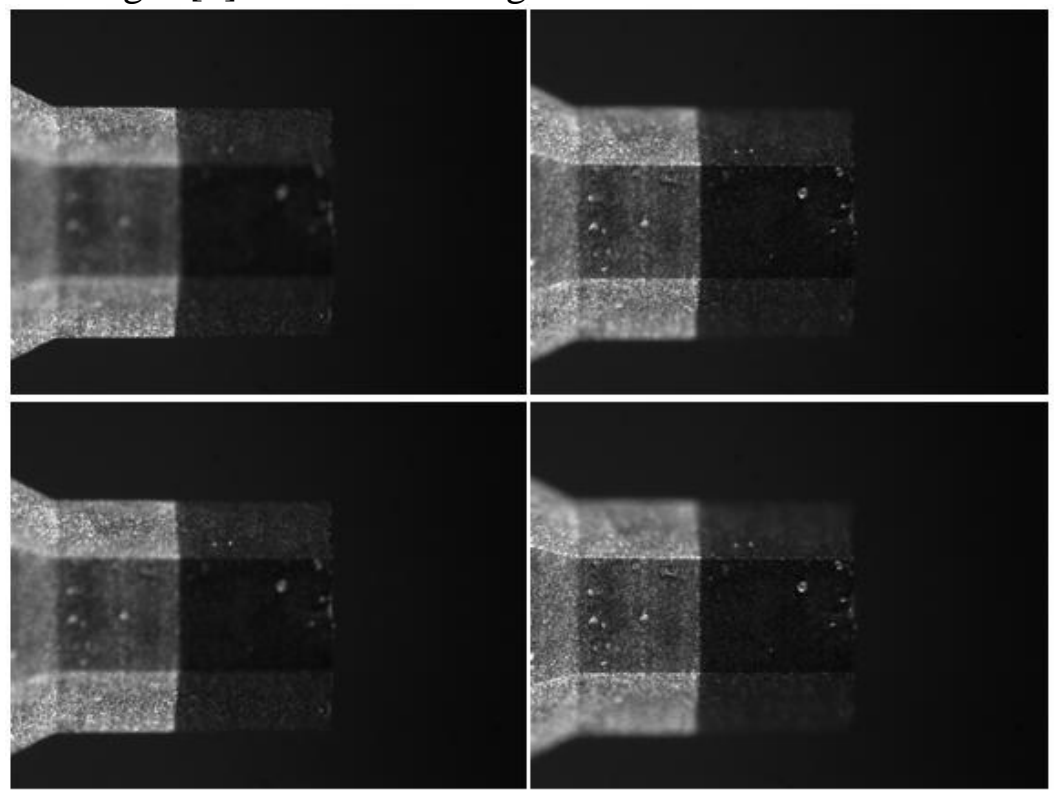

Fig. 5 Multi focus image of micro tool observation

In recent years, image fusion algorithm based on focusing evaluation function has been widely used in image fusion, and has got a great success. In numerous of focusing evaluation function, the grey function has a better performance which can fuse image easily and quickly [10]. The common focus evaluation functions include variance, energy of image gradient, laplacian, sobel-tenengrad function, etc. The multi-focus image fusion based on variance function is selected in this article, whose calculation speed is faster other algorithms. The fusion time is about $1.5 \mathrm{~s}$, which can meet the requirements of the on-line wear measurement completely. Fig. 6 shows the image fusion results of two kinds of typical micro tool from five images.

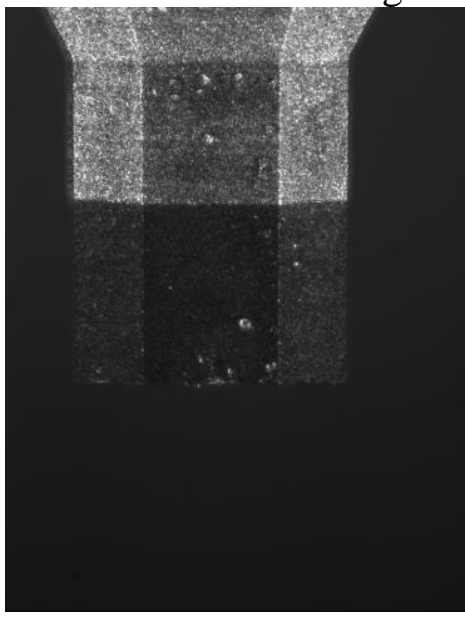

(a) Hexagon end mill

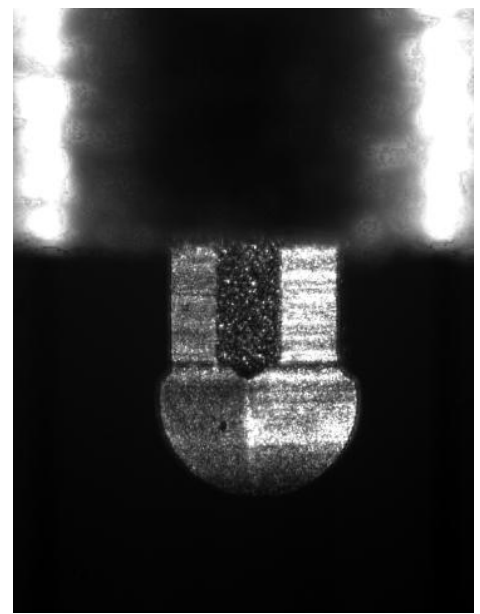

(b) Ball end mill

Fig. 6 Micro tool wear of front light observation image fusion

\section{End Surface Observation of Micro Tool}

Micro milling depth and feed rate is very small in micro parts processing, so that the cutting force per unit area is large, which will produce high heat at the same time and raise the temperature of the micro tool tip region. Therefore, micro milling cutter wear mainly occurred at the end of the cutting tool compared with regular size of the milling cutter wear mainly occurs in the blade surface. At present, there are few research about the end surface observation of micro tool in the academia. In this paper, the end surface image can be observed directly by the developed system. Also through the above variance evaluation function, the multi-focus image of the terminal image can be fused, so that the 
operators can get the characteristics and wear pattern on the end of micro tools. The two end surface images of two typical micro tools are shown in Fig. 7.

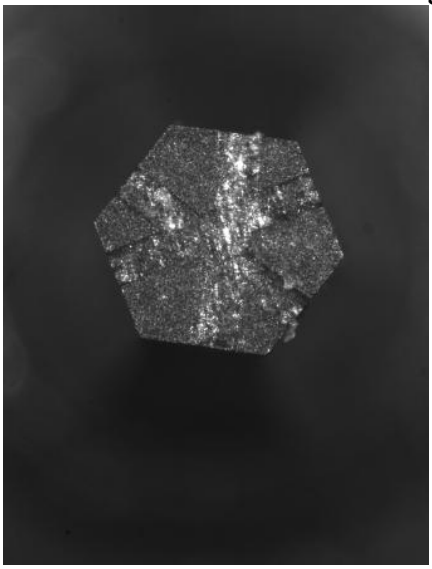

(a) Hexagon end mill

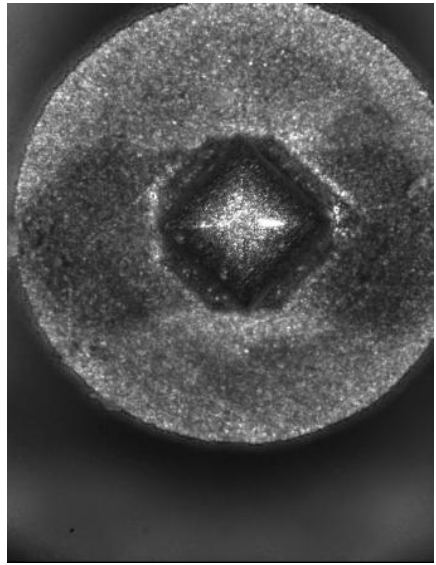

(b) Ball end mill

Fig. 7 Terminal image fusion and wear observation

\section{Summary}

This paper investigated the on-line tool inspection measurement method based on machine vision, which have some obvious advantages such as high reliability, automation, non-contact, high accuracy, etc. Through observing the on-line wear conditions, the operators can optimize the machining parameters to improve the machining efficiency and accuracy of micro-milling. The measurement experiments on-machine tool verified that the proposed methods as well as the developed measurement system can basically meet the needs of industrial applications.

\section{Acknowledgements}

The authors would like to express their sincere appreciation to National Natural Science Foundation of China (Grant No. 51205243 and Grant No. 41376169), Research Fund for the Doctoral Program of Higher Education of China (Grant No. 20123108120009) for supporting this research.

\section{References}

[1] S.Gopi Krishna, V.R.Raju, Manufacture of micro sized threads for micro actuator of laser grating mount, The International Journal of Advanced Manufacturing Technology, 61(2012), 1215-1220.

[2] A. M. Hey, W.M.P. Gomez, J. E. Ruzzante. Tool wear evaluation in drilling by acoustic emission, Physics Procedia. 3 (2010) 819-825.

[3] X.Q. Chen, H.Z. Li. Development of a tool wear observer model for online tool condition monitoring and control. Int J Adv Manuf Technol. 45(2009) 786-800.

[4] D. A. Fadare, A.O. Oni. Development and application of machine vision system for measurement of tool wear.RPN Journal of Engineering and Applied Sciences, 4(2009) 42-49.

[5] Information on http://www.big-daishowa.co.jp

[6] C. Steger, M. Ulrich, C. Wiedemann, Machine Vision Algorithms and Applications, first ed., Beijing, 2008.

[7] A. Archenti, M. Nicolescu, A New Method for Circular Testing of Machine Tools Under Loaded Condition, Procedia CIRP, 1(2012), , 575-580.

[8] S.K.Nayar, Y.Nakagawa. Shape from focus. IEEE Transactions on Pattern Analysis and Machine Intelligence. 16(1994) 824-831.

[9] W.Huang, Z.Jing, Evaluation of focus measures in multi-focus image fusion. Pattern Recogn Letters., 28(2007) 493-500.

[10] G. Piella. A general framework for multi resolution image fusion: from pixels to regions, Information fusion, 4(2003) 259-280. 